\title{
IMBALANCED STRONG MHD TURBULENCE
}

\author{
Y. Lithwick, ${ }^{1}$ P. Goldreich, ${ }^{2}$ and S. SRIDHar ${ }^{3}$ \\ Received 2006 July 17; accepted 2006 October 4
}

\begin{abstract}
We present a phenomenological model of imbalanced MHD turbulence in an incompressible magnetofluid. The steady state cascades, of waves traveling in opposite directions along the mean magnetic field, carry unequal energy fluxes to small length scales, where they decay as a result of viscous and resistive dissipation. The inertial range scalings are well understood when both cascades are weak. We study the case in which both cascades are, in a sense, strong. The inertial range of this imbalanced cascade has the following properties: (1) The ratio of the rms Elsässer amplitudes is independent of scale and is equal to the ratio of the corresponding energy fluxes. (2) In common with the balanced strong cascade, the energy spectra of both Elsässer waves are of the anisotropic Kolmogorov form, with their parallel correlation lengths equal to each other on all scales, and proportional to the two-thirds power of the transverse correlation length. (3) The equality of cascade time and wave period (critical balance) that characterizes the strong balanced cascade does not apply to the Elsässer field with the larger amplitude. Instead, the more general criterion that always applies to both Elsässer fields is that the cascade time is equal to the correlation time of the straining imposed by oppositely directed waves. (4) In the limit of equal energy fluxes, the turbulence corresponds to the balanced strong cascade. Our results are particularly relevant for turbulence in the solar wind. Spacecraft measurements have established that in the inertial range of solar wind turbulence, waves traveling away from the Sun have higher amplitudes than those traveling toward it. Result 1 allows us to infer the turbulent flux ratios from the amplitude ratios, thus providing insight into the origin of the turbulence.
\end{abstract}

Subject headings: MHD - turbulence

\section{INTRODUCTION}

Magnetohydrodynamic (MHD) turbulence is present in many astronomical settings, such as the solar wind, the interstellar medium, molecular clouds, accretion disks, and clusters of galaxies (Biskamp 2003; Kulsrud 2005; Schekochihin \& Cowley 2007). Its theory has attracted a sizable literature (Iroshnikov 1963; Kraichnan 1965; Shebalin et al. 1983; Goldreich \& Sridhar 1995, 1997; Ng \& Bhattacharjee 1996; Cho \& Vishniac 2000; Biskamp \& Müller 2000; Maron \& Goldreich 2001; Cho et al. 2002; Galtier et al. 2000, 2002; Müller et al. 2003; Galtier et al. 2005; Boldyrev 2005; Müller \& Grappin 2005; Beresnyak \& Lazarian 2006). The simplest of cases concerns the small-scale dynamics of the excitations of an incompressible fluid with a mean magnetic field. The turbulent cascade of energy to small scales is the result of nonlinear interactions between Alfvén waves traveling in opposite directions along the local, mean magnetic field (Iroshnikov 1963; Kraichnan 1965). Whereas this broad picture of Iroshnikov and Kraichnan still endures, our appreciation of MHD turbulence has undergone significant revisions due, mainly, to the recognition of the importance of anisotropy and the consequent strengthening of nonlinear interactions. The inertial range, which includes length scales between the stirring and dissipation scales, is best understood for those cases in which the oppositely directed waves are excited with equal power; these balanced cascades can be weak (Ng \& Bhattacharjee 1996; Goldreich \& Sridhar 1997), as well as strong (Goldreich \& Sridhar 1995). Imbalanced cascades are understood only for the case in which the turbulence is weak (Galtier et al. 2000; Lithwick \& Goldreich 2003). In this paper, we consider the general case of imbalanced cascades.

\footnotetext{
${ }^{1}$ Canadian Institute for Theoretical Astrophysics, Toronto, ON, Canada; yoram@cita.utoronto.ca.

2 Institute for Advanced Study, Princeton, NJ; pmg@ias.edu.

3 Raman Research Institute, Bangalore, India; ssridhar@rri.res.in.
}

The solar wind is the best laboratory that we have to investigate MHD turbulence. In situ measurements by spacecraft have yielded spectra for velocity and magnetic field fluctuations over many decades of length scale (e.g., Horbury 1999). On the largest scales the spectrum is flat, presumably reflecting the spectrum with which fluctuations are injected into the solar wind by shocks or dynamical instabilities. On smaller scales, the spectrum is Kolmogorov, and fluctuations are thought to be undergoing an active turbulent cascade. On these scales, the amplitudes of the two Elsässer fields are not equal: waves traveling away from the Sun have higher amplitudes than those traveling toward it. ${ }^{4}$ Because of this imbalance, the theory of MHD turbulence has been inadequate for application to the solar wind. Our solution for the strong imbalanced cascade removes this inadequacy.

In $\S 2$, we summarize the properties of MHD cascades that were previously understood. Our solution for the strong imbalanced cascade is given in $\S 3$.

\section{BALANCED CASCADES AND THE IMBALANCED WEAK CASCADE}

The system we consider is an incompressible magnetofluid of mass density $\rho$ and mean magnetic field $B_{0} \hat{z}$. Let $\boldsymbol{v}(\boldsymbol{r}, t)$ be the fluid velocity and $\boldsymbol{b}(\boldsymbol{r}, t)$ the magnetic field fluctuation. The MHD equations determining their time evolution are expressed conveniently in terms of the Elsässer fields, ${ }^{5} \boldsymbol{w}^{ \pm}=\boldsymbol{v} \mp \boldsymbol{b} /(4 \pi \rho)^{1 / 2}$ :

$$
\left(\partial_{t} \pm V_{\mathrm{A}} \partial_{z}\right) \boldsymbol{w}^{ \pm}+\left(\boldsymbol{w}^{\mp} \cdot \nabla\right) \boldsymbol{w}^{ \pm}=-\nabla(p / \rho)
$$

where $V_{\mathrm{A}}=B_{0} /(4 \pi \rho)^{1 / 2}$ is the Alfvén speed and $p$ is the total pressure, determined by requiring $\nabla \cdot \boldsymbol{w}^{ \pm}=0$. We have neglected

\footnotetext{
${ }^{4}$ This imbalance is more pronounced closer to the Sun.

5 The superscripts on $\boldsymbol{w}^{+}$and $\boldsymbol{w}^{-}$refer to the direction of wave propagation.
} 


\section{Before Collision}

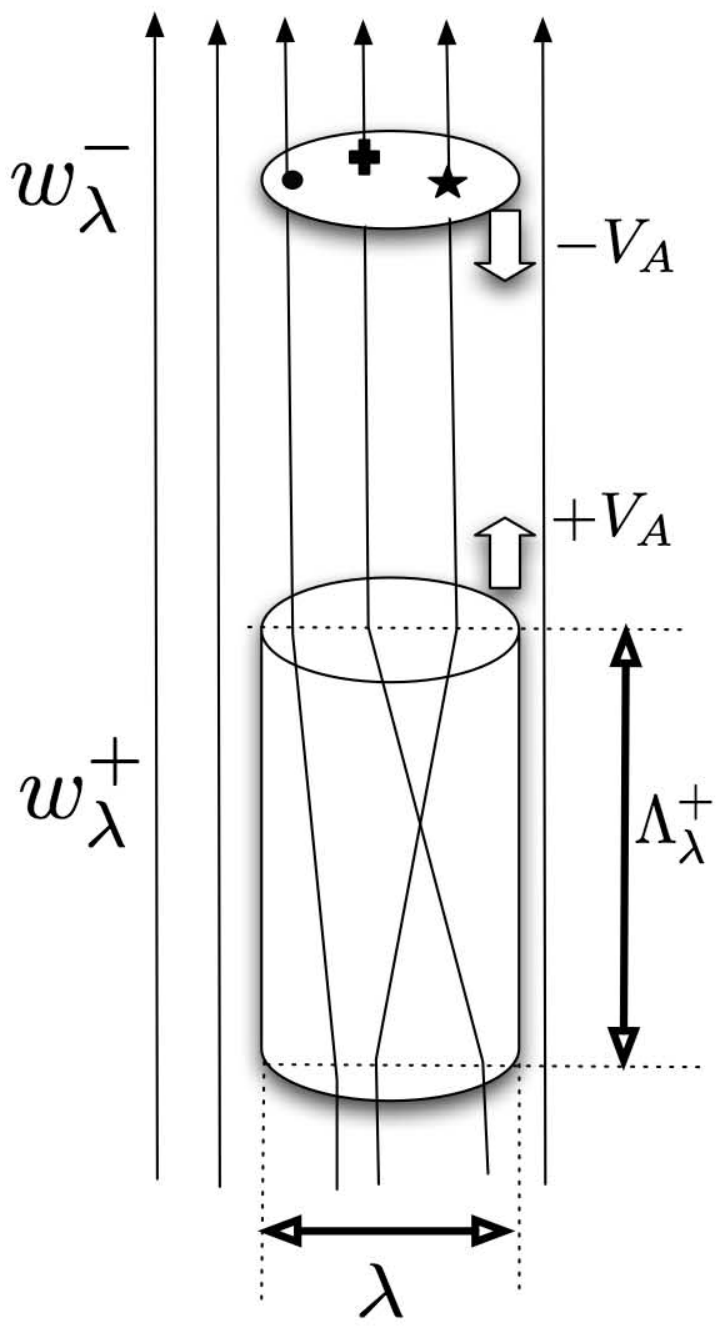

\section{After Collision}

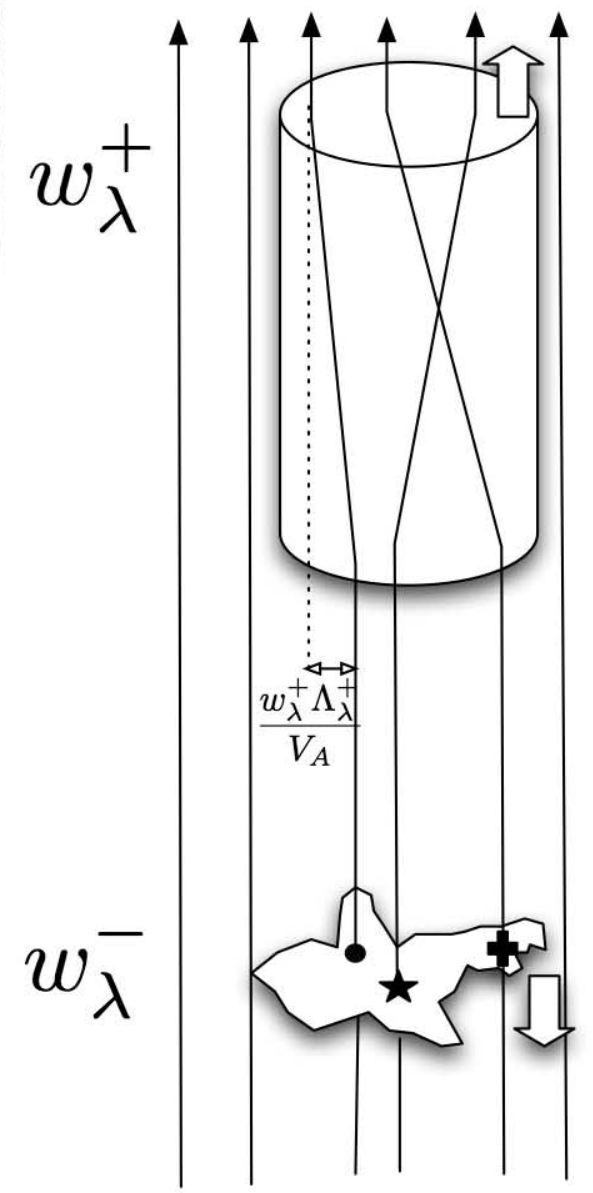

FIG. 1.-Collision between a wave packet and a slice. The mostly vertical lines are magnetic field lines. Before the collision, a localized wave packet of $w_{\lambda}^{+}$, with transverse

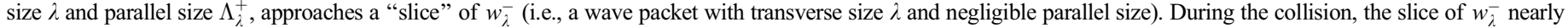
follows the perturbed field lines within the up-going wave packet. Hence it is distorted after the collision.

viscous and resistive dissipative terms, which are important on small scales; we have also neglected forcing terms, which are important on large scales. When either $\boldsymbol{w}^{+}$or $\boldsymbol{w}^{-}$is initially zero everywhere, the nonlinear term vanishes for all time. Then, either $\left\{\boldsymbol{w}^{-}=\boldsymbol{w}^{-}\left(x, y, z+V_{\mathrm{A}} t\right), \boldsymbol{w}^{+}=0\right\}$ or $\left\{\boldsymbol{w}^{+}=\boldsymbol{w}^{+}\left(x, y, z-V_{\mathrm{A}} t\right)\right.$, $\left.\boldsymbol{w}^{-}=0\right\}$ is a nonlinear solution of arbitrary form that propagates in the $-\hat{z}$ or $+\hat{z}$ direction with speed $V_{\mathrm{A}}$. Kraichnan recognized that the existence of these nonlinear solutions implies that MHD turbulence can be described as interactions between oppositely directed wave packets. Equation (1) conserves the Elsässer energies, $E^{ \pm}=\frac{1}{2} \int\left|\boldsymbol{w}^{ \pm}\right|^{2} d^{3} x$, and hence collisions between wave packets do not lead to exchanges between $E^{+}$and $E^{-}$, but only to a redistribution of the energies over different length scales.

Interactions between oppositely directed wave packets are best understood geometrically. The magnetic field perturbation within a wave packet of $\boldsymbol{w}^{+}$, say, bends the background magnetic field lines (Fig. 1). When a thin "slice" of $\boldsymbol{w}^{-}$passes through the up- going wave packet, it approximately follows - i.e., is advected by - the total (perturbed plus unperturbed) magnetic field lines induced by the $\boldsymbol{w}^{+}$. Therefore, oppositely directed wave packets distort one another. When a wave packet suffers an order-unity distortion, its energy can be considered to have cascaded to a smaller length scale.

To be quantitative, consider an up-going wave packet that has size $\lambda$ transverse to the mean field, size $\Lambda_{2}^{+}$parallel to the mean field, and a value for $\boldsymbol{w}^{+}$that varies by order unity across the wave packet, with typical amplitude $\sim w_{\lambda}^{+}$. Since the magnetic field perturbation within the up-going wave packet is $\sim B_{0} w_{\lambda}^{+} / V_{\mathrm{A}}$, field lines on opposite sides of the wave packet are inclined by a relative angle $\theta_{\lambda} \sim w_{\lambda}^{+} / V_{\mathrm{A}}$. Therefore, the field lines' separations through the wave packet change by $\Lambda_{\lambda}^{+} \theta_{\lambda} \sim \Lambda_{\lambda}^{+} w_{\lambda}^{+} / V_{\mathrm{A}}$ (Fig. 1). The down-going slice cascades to smaller scales when the separations between the field lines it is following suffer order-unity changes. Since we consider only a thin slice of $w_{\lambda}^{-}$, the backreaction on the $w_{\lambda}^{+}$wave packet can be neglected. There are two 
possibilities for the slice's cascade time, depending on the amplitude of the wave packet's dimensionless parameter

$$
\chi_{\lambda}^{+} \equiv \frac{\Lambda_{\lambda}^{+} w_{\lambda}^{+}}{\lambda V_{\mathrm{A}}}
$$

If $\chi_{\lambda}^{+}>1$, the slice will cascade after it has traveled a distance $\lambda / \theta_{\lambda} \sim \lambda V_{\mathrm{A}} / w_{\lambda}^{+}<\Lambda_{\lambda}^{+}$through the wave packet. Since the relative speed of slice and wave packet is $2 V_{\mathrm{A}}$, the slice's cascade time is $\sim \lambda / w_{\lambda}^{+}$.

Conversely, if $\chi_{\lambda}^{+}<1$, the slice will be weakly distorted after crossing a single wave packet. It will only cascade after colliding with more up-going wave packets. Assuming these all have similar size $\left(\lambda, \Lambda_{\lambda}^{+}\right)$and amplitude $\left(w_{\lambda}^{+}\right)$, although with random orientation of $\boldsymbol{w}^{+}$, the cascade occurs in a random-walk fashion: since each wave packet distorts the slice by a fractional amount $\chi_{\lambda}^{+}$, the slice cascades after crossing $N \sim\left(\chi_{\lambda}^{+}\right)^{-2}$ wave packets. Multiplying by the time to cross a single wave packet, $\Lambda_{\lambda}^{+} / V_{\mathrm{A}}$, yields the slice's cascade time, $\left(\chi_{\lambda}^{+}\right)^{-2} \Lambda_{\lambda}^{+} / V_{\mathrm{A}}$.

Summarizing the preceding two paragraphs, the down-going slice cascades in a time

$$
\tau_{\lambda}^{-} \sim \begin{cases}\lambda / w_{\lambda}^{+}, & \text {if } \chi_{\lambda}^{+}>1 \\ \left(\lambda / w_{\lambda}^{+}\right)\left(\chi_{\lambda}^{+}\right)^{-1}, & \text { if } \chi_{\lambda}^{+}<1\end{cases}
$$

In a turbulent cascade, the $\boldsymbol{w}^{ \pm}$fields vary over many length scales. We may consider these fields to be a superposition of many wave packets. On each transverse length scale $\lambda$, wave packets have typical amplitude $w_{\lambda}^{ \pm}$and a typical parallel size $\Lambda_{\lambda}^{ \pm}$, where $\Lambda_{\lambda}^{ \pm}$can be scale dependent; it is typically much larger than $\lambda$. These quantities can be defined more formally, with $w_{\lambda}^{ \pm}$defined as the rms $\boldsymbol{w}^{ \pm}$ difference between two points separated by transverse scale $\lambda$ and $\Lambda_{\lambda}^{ \pm}$the scale along the mean field such that, given $\lambda$ and $w_{\lambda}^{ \pm}$, the rms $\boldsymbol{w}^{ \pm}$difference between two points separated by parallel distance $\Lambda_{\lambda}^{ \pm}$is also $w_{\lambda}^{ \pm}$(e.g., Lithwick \& Goldreich 2001). However, for the purpose of deriving the turbulent scalings, such rigor is not required. In addition, when we refer to the direction of the mean field, it should be borne in mind that on a given length scale, fluctuations on all larger length scales contribute to the local mean field; it is the direction of this local mean field that is relevant (Cho \& Vishniac 2000; Maron \& Goldreich 2001).

Although it is more common to describe turbulence in Fourier space, in this paper we choose to describe it in real space, in terms of interactions between wave packets. A more precise treatment would likely require the computation of interactions between wave modes in Fourier space. For the purpose of comparing the two descriptions, $\lambda \sim k_{\perp}^{-1}, \Lambda_{\lambda}^{ \pm} \sim\left(k_{\|}^{ \pm}\right)^{-1}$, and $w_{\lambda}^{ \pm} \sim A k_{\perp}\left(k_{\|}^{ \pm}\right)^{1 / 2}\left|\hat{w}_{k}^{ \pm}\right|$, where $\left|\hat{w}_{k}^{ \pm}\right|$is the amplitude of the Fourier mode, $A$ is a normalization constant, and we use the standard notation $k_{\perp}, k_{\|}$for the wavenumbers perpendicular and parallel to the mean field, though here we distinguish the $k_{\|}$'s for the positive and negative waves with superscripts.

The value of

$$
\chi_{\lambda}^{ \pm} \equiv \frac{\Lambda_{\lambda}^{ \pm} w_{\lambda}^{ \pm}}{\lambda V_{\mathrm{A}}}
$$

influences the inertial range scalings of physical quantities. Below we provide a brief review of the cases that are understood to some extent; this enables us to pose our question more sharply. In the balanced cases, we set $w_{\lambda}^{+}=w_{\lambda}^{-}=w_{\lambda}$ and $\Lambda_{\lambda}^{+}=\Lambda_{\lambda}^{-}=\Lambda_{\lambda}$, which implies that $\chi_{\lambda}^{+}=\chi_{\lambda}^{-}=\chi_{\lambda}=\Lambda_{\lambda} w_{\lambda} / \lambda V_{\mathrm{A}}$.

1. Balanced weak turbulence, $\chi_{\lambda} \ll 1$. - The turbulent cascade is due to resonant three-wave interactions $(\mathrm{Ng} \&$ Bhattacharjee 1996; Goldreich \& Sridhar 1997; Galtier et al. 2000; Lithwick \& Goldreich 2003). The rms amplitude across a transverse scale $\lambda$ is $w_{\lambda} \propto \lambda^{1 / 2}$. Frequency resonance conditions prevent a parallel cascade, so the parallel scale is independent of $\lambda$; its value is set by conditions at the stirring scale. Perturbation theory is valid when $\chi_{\lambda} \ll 1$ and can be used to derive kinetic equations describing the inertial range. However, the cascade strengthens on small scales, because $\chi_{\lambda} \propto 1 / \lambda^{1 / 2}$ increases as $\lambda$ decreases; this limits the validity of perturbation theory, and the inertial range of weak turbulence.

2. Balanced strong turbulence, $\chi_{\lambda} \sim 1$.-Perturbation theory is inapplicable. According to the phenomenological theory of Goldreich \& Sridhar (1995), the strength of the interactions remains of order unity: $\chi_{\lambda} \sim 1$ ("critical balance"). The cascade is of anisotropic Kolmogorov form, with $w_{\lambda} \propto \lambda^{1 / 3}$ and $\Lambda_{\lambda} \propto \lambda^{2 / 3}$. Turbulence that is excited with $\chi_{\lambda}>1$ weakens to $\chi_{\lambda} \sim 1$ in less than the Alfvén crossing time.

3. Imbalanced weak turbulence, $\chi_{\lambda}^{ \pm} \ll 1$. - In common with the balanced case, the process can be described in detail, using the weak-turbulence theory of resonant three-wave interactions (Galtier et al. 2000; Lithwick \& Goldreich 2003). As before, the product $w_{\lambda}^{+} w_{\lambda}^{-} \propto \lambda$, and frequency resonance conditions forbid a parallel cascade, so the two parallel scales $\Lambda_{\lambda}^{ \pm}$are scale independent. However, in contrast to the balanced case, $(a)$ kinetic equations are required to relate the spectral indices of the oppositely directed waves to the ratio of their fluxes, and $(b)$ the ratio of $w_{\lambda}^{+}$to $w_{\lambda}^{-}$in the inertial range depends upon the ratio of $\lambda$ to the dissipation scale, $\lambda_{\text {diss }}$, since the two amplitudes are forced to equal one another at $\lambda_{\text {diss }}$.

As discussed in Lithwick \& Goldreich (2003), when the dissipation scale is large enough (while still remaining smaller than the stirring scale), imbalanced turbulence can be weak throughout the inertial range. However, both $\chi_{\lambda}^{+}$and $\chi_{\lambda}^{-}$increase with decreasing $\lambda$, thereby limiting the inertial range. Therefore, in the physically important limit of very small dissipation, it is inevitable that at least one of the $w_{\lambda}^{+}$or $w_{\lambda}^{-}$cascades becomes strong.

\section{THE IMBALANCED STRONG CASCADE}

In the present section, the heart of this paper, we derive the spectrum of imbalanced strong turbulence. We assume that $w_{\lambda}^{+} \gg$ $w_{\lambda}^{-}$. This assumption is made only for clarity of presentation. Our discussion is more generally valid, for all $w_{\lambda}^{+} \geq w_{\lambda}^{-}$. Our derivation is based on the physical picture described above: negative waves $\left(w_{\lambda}^{-}\right)$nearly follow field lines perturbed by positive waves $\left(w_{\lambda}^{+}\right)$, and vice versa. To calculate the spectra, we need the cascade times of the down- and up-going waves, which can then be substituted into the Kolmogorov scaling relations (eqs. [12]-[13]).

Let $\chi_{\lambda}^{+} \geq 1$. Otherwise, the turbulence would be weak. Consider a packet of positive waves that crosses a packet of negative waves, both having transverse scale $\lambda$. The cascade time of $w_{\lambda}^{-}$ (eq. [3]) is

$$
\tau_{\lambda}^{-} \sim \lambda / w_{\lambda}^{+}
$$

We now calculate the parallel size of a negative wave packet. Consider the $\boldsymbol{w}^{-}$field in a plane with $z+V_{\mathrm{A}} t=$ const, that is, in a plane that is transverse to the mean field, and that is comoving with $\boldsymbol{w}^{-}$, traveling downward at speed $V_{\mathrm{A}}$. After the plane travels a distance equal to the cascade distance of $w_{\lambda}^{-}$(cascade 
distance $V_{\mathrm{A}} \tau_{\lambda}^{-}$), the $w_{\lambda}^{-}$within the plane will suffer distortions of order unity. Therefore, two planes of $w_{\lambda}^{-}$that are separated by a parallel distance greater than the cascade distance will evolve independently and consequently must be uncorrelated. It follows that a $w_{2}^{-}$wave packet has parallel size

$$
\Lambda_{\lambda}^{-} \sim V_{\mathrm{A}} \tau_{\lambda}^{-} \sim\left(V_{\mathrm{A}} / w_{\lambda}^{+}\right) \lambda
$$

Since this relation mixes $\Lambda_{\lambda}^{-}$and $w_{\lambda}^{+}$, it does not amount to critical balance for either positive or negative waves.

The parallel size of $w_{\lambda}^{+}$wave packets follows from a thought experiment analogous to the one described above: Consider two planes of $\boldsymbol{w}^{+}$, separated by a distance $D$ and moving upward at speed $V_{\mathrm{A}}$. If $D \gg \Lambda_{\lambda}^{-}$, the positive waves in the leading plane will encounter negative waves that are completely different from the negative waves encountered in the trailing plane. If $D \ll \Lambda_{\lambda}^{-}$, then the negative waves that cascade the leading $w_{\lambda}^{+}$are almost the same as those that cascade the trailing $w_{\lambda}^{+}$. The critical $D$ is clearly $\Lambda_{\lambda}^{-}$. Therefore,

$$
\Lambda_{\lambda}^{+} \sim \Lambda_{\lambda}^{-}
$$

This can also be understood as follows: Consider a wave packet of $w_{\lambda}^{+}$with very large $\Lambda_{\lambda}^{+}$that interacts with a train of negative wave packets, each with parallel size $\Lambda_{\lambda}^{-} \ll \Lambda_{\lambda}^{+}$. Then any two regions within the up-going wave packet that are separated by a parallel distance greater than $\Lambda_{\lambda}^{-}$will be cascaded by statistically uncorrelated negative waves, in which case the $w_{\lambda}^{-}$imprint their parallel scales on the $w_{\lambda}^{+}$. Henceforth we denote the common parallel scales by $\Lambda_{\lambda}$. This discovery that the parallel scales are similar in an imbalanced cascade is a nontrivial result. Equations (6) and (7) yield

$$
\chi_{\lambda}^{+}=\frac{w_{\lambda}^{+} \Lambda_{\lambda}}{V_{\mathrm{A}} \lambda} \sim 1
$$

proving that the cascade of negative waves is critically balanced.

It remains to calculate the positive waves' cascade time. All of the following material up to equation (11), as well as the material in the Appendix, is devoted to deriving the result: $\tau_{\lambda}^{+} \sim \lambda / w_{\lambda}^{-}$. This result is remarkable. It shows that the straining rate imposed by the negative waves on positive ones, $w_{\lambda}^{-} / \lambda$, is imposed coherently over a time $\lambda / w_{\lambda}^{-}$. Yet the wave period of negative waves is much shorter than this, by the factor

$$
\chi_{\lambda}^{-}=\frac{w_{\lambda}^{-} \Lambda_{\lambda}}{V_{\mathrm{A}} \lambda} \sim \frac{w_{\lambda}^{-}}{w_{\lambda}^{+}} \ll 1 .
$$

Since $\chi_{\lambda}^{-} \ll 1$, one might be tempted to conclude, erroneously, that positive waves undergo a weak cascade, that is, that negative waves impose on them small, short, uncorrelated strains of amplitude $\chi_{\lambda}^{-}$over time intervals $\sim \Lambda_{\lambda} / V_{\mathrm{A}}$, resulting in $\tau_{\lambda}^{+} \sim$ $\left(\chi_{\lambda}^{-}\right)^{-2} \Lambda_{\lambda} / V_{\mathrm{A}} \sim\left(\chi_{\lambda}^{-}\right)^{-1} \lambda / w_{\lambda}^{-}$(as in eq. [3]). Instead, the correct conclusion is that a coherent strain is imposed over a time interval $\lambda / w_{\lambda}^{-}$.

How can the coherence time exceed $\Lambda_{\lambda} / V_{\mathrm{A}}$ ? The key point is that the straining of the positive waves is due to the $\boldsymbol{w}^{-}$field as seen from the positive waves' rest frame (which has $x^{\prime}=x, y^{\prime}=y$, $z^{\prime}=z-V_{\mathrm{A}} t, t^{\prime}=t$ ). In this frame, the MHD equations (eq. [1]) transform to

$$
\begin{gathered}
\partial_{t^{\prime}} \boldsymbol{w}^{+}+\left(\boldsymbol{w}^{-} \cdot \nabla^{\prime}\right) \boldsymbol{w}^{+}=-\nabla^{\prime}(p / \rho), \\
\left(\partial_{t^{\prime}}-2 V_{\mathrm{A}} \partial_{z^{\prime}}\right) \boldsymbol{w}^{-}+\left(\boldsymbol{w}^{+} \cdot \nabla^{\prime}\right) \boldsymbol{w}^{-}=-\nabla^{\prime}(p / \rho) .
\end{gathered}
$$

To appreciate that the correlation time of $\boldsymbol{w}^{-}$in the primed frame can exceed $\Lambda_{\lambda} / V_{\mathrm{A}}$, consider the limiting case in which $\boldsymbol{w}^{-}$is so small that back-reaction onto the positive waves can be neglected. Then $\boldsymbol{w}^{+}$is independent of $t^{\prime}$ and is a function only of $\boldsymbol{r}^{\prime}$, so $\boldsymbol{w}^{-}$satisfies a linear (integrodifferential) equation, whose coefficients are independent of $t^{\prime}$. If negative waves are injected on a length scale much larger than the scale of interest, with a long coherence time $T$, then as they cascade down to smaller scales their coherence time remains fixed, $\tau_{\text {corr }, \lambda}^{-}=T$, where $\tau_{\text {corr }, \lambda}^{-}$is defined as the correlation time of the negative waves in the frame of the positive waves. In the limiting case that $\boldsymbol{w}^{-}$is held fixed at the injection scale $(T=\infty)$, then on smaller scales $\boldsymbol{w}^{-}$is independent of $t^{\prime}\left(\tau_{\text {corr }, \lambda}^{-}=\infty\right)$, even though it is undergoing an active cascade to small scales.

To estimate $\tau_{\text {corr, } \lambda}^{-}$when $\boldsymbol{w}^{-}$is not infinitesimally small, it is necessary to account for back-reaction: negative waves alter positive waves, which react back onto the negative ones. As negative waves cross a plane at fixed $z^{\prime}$, the positive waves at that plane are changing on their cascade timescale $\tau_{\lambda}^{+}$. Hence, over times separated by $\tau_{\lambda}^{+}$, the negative waves crossing $z^{\prime}$ are cascaded by entirely different positive waves. This implies that $\tau_{\text {corr }, \lambda}^{-} \sim \tau_{\lambda}^{+}$. Because the positive waves are strained at the rate $w_{\lambda}^{-} / \lambda$, it follows that

$$
\tau_{\lambda}^{+} \sim \lambda / w_{\lambda}^{-}
$$

Invoking Kolmogorov's hypothesis of the scale (i.e., $\lambda$ ) independence of the energy fluxes,

$$
\begin{aligned}
& \epsilon^{-} \sim \frac{\left(w_{\lambda}^{-}\right)^{2}}{\tau_{\lambda}^{-}} \sim \frac{\left(w_{\lambda}^{-}\right)^{2} w_{\lambda}^{+}}{\lambda}, \\
& \epsilon^{+} \sim \frac{\left(w_{\lambda}^{+}\right)^{2}}{\tau_{\lambda}^{+}} \sim \frac{\left(w_{\lambda}^{+}\right)^{2} w_{\lambda}^{-}}{\lambda},
\end{aligned}
$$

we obtain the inertial range scalings,

$$
\begin{gathered}
w_{\lambda}^{ \pm} \sim \frac{\left(\epsilon^{ \pm}\right)^{2 / 3}}{\left(\epsilon^{\mp}\right)^{1 / 3}} \lambda^{1 / 3} ; \\
\Lambda_{\lambda} \sim \frac{\left(\epsilon^{-}\right)^{1 / 3}}{\left(\epsilon^{+}\right)^{2 / 3}} V_{\mathrm{A}} \lambda^{2 / 3} .
\end{gathered}
$$

The positive-wave cascade shares some characteristics with both weak and strong balanced MHD cascades. In the weak cascades, the cascade time is longer than the wave period, and a wave experiences multiple, randomly phased perturbations during its cascade time. In the strong cascades, the cascade time is comparable to (or shorter than) the wave period, and a wave suffers a coherent strain as it cascades. Furthermore, weak turbulence submits to perturbation theory but strong turbulence does not. In the positive-wave cascade,

1. The period of a positive wave is shorter than its cascade time;

2. A positive wave is coherently strained as it cascades $;{ }^{7}$ and

3. The positive-wave cascade is nonperturbative.

We contend that the positive-wave cascade is strong because the second and third items have dynamical significance whereas the

\footnotetext{
6 We are implicitly assuming that most of the change in $w_{\lambda}^{-}$is accumulated on scales comparable to $\lambda$. We justify this assumption quantitatively in the Appendix.

7 The correlation time of the strain induced by the negative waves, $\tau_{\text {corr }, \lambda}^{-}$, is comparable to the cascade time, $\tau_{\lambda}^{+}$, of the positive waves.
} 
first does not. The dimensionless parameter that indicates whether the positive waves are strongly cascaded is

$$
\hat{\chi}_{\lambda}^{-} \equiv w_{\lambda}^{-} \tau_{\text {corr }, \lambda}^{-} / \lambda
$$

and not $\chi_{\lambda}^{-}$. Strong cascades correspond to $\hat{\chi}_{\lambda}^{-} \sim 1$ and weak ones to $\hat{\chi}_{\lambda}^{-}<1$. For the negative-wave cascade $\hat{\chi}_{\lambda}^{+}=\chi_{\lambda}^{+} \sim 1$, since the correlation time of positive waves in the frame of the negative ones is $\Lambda_{\lambda} / V_{\mathrm{A}}$. We call the criterion $\hat{\chi}_{\lambda}^{ \pm} \sim 1$ "modified critical balance," to distinguish it from critical balance (which would incorrectly imply $\chi_{\lambda}^{ \pm} \sim 1$ ).

It is interesting to note that equations (12)-(13) are the same as might have been guessed directly from the MHD equations (eq. [1]) by simply replacing $\partial_{t} \boldsymbol{w}^{ \pm} \rightarrow w_{\lambda}^{ \pm} / \tau_{\lambda}^{ \pm}$and $\left(\boldsymbol{w}^{\mp} \cdot \nabla\right) \boldsymbol{w}^{ \pm} \rightarrow$ $w_{\lambda}^{\mp} w_{\lambda}^{ \pm} / \lambda$ and dropping the other two terms. In fact, equations (12)(13) appear in Verma et al. (1996) and Verma (2004), although those papers assume isotropy, whereas in our derivation anisotropy plays a fundamental role. To test our theory with either numerical simulations or solar wind observations, one need verify not only equation (14), but the anisotropy relation (eq. [15]) as well. An even more compelling test would be to check that $\hat{\chi}_{\lambda}^{ \pm} \sim 1$ throughout the inertial range.

Politano \& Pouquet (1998a, 1998b) derived general relations for triple correlation functions in MHD turbulence. ${ }^{8}$ If one cavalierly pulls the individual factors of $w^{+}$and $w^{-}$out of their correlations, then our equations (12)-(13) are recovered. Politano \& Pouquet's relations are intriguing, since it is not obvious how they depend upon the elongation of wave packets about the mean field, which is an essential element of our theory.

\section{SUMMARY}

We have deduced the behavior of imbalanced strong MHD turbulence. Its salient properties are as follows:

1. The positive and negative waves carry unequal energy fluxes, $\epsilon^{+} \neq \epsilon^{-}$, while they both undergo strong cascades.

\footnotetext{
${ }^{8}$ We thank M. Forman for pointing out this reference to us.
}

2. In the inertial range, the rms Elsässer amplitudes are proportional to the one-third power of the transverse scale: $w_{\lambda}^{ \pm} \propto \lambda^{1 / 3}$. This is similar to the balanced strong cascade. Moreover, their ratio, $w_{\lambda}^{+} / w_{\lambda}^{-} \sim \epsilon^{+} / \epsilon^{-}$, is independent of $\lambda$.

3. The parallel scales of the positive and negative waves are equal. The common parallel scale of eddies of transverse scale $\lambda$ is $\Lambda_{\lambda} \propto \lambda^{2 / 3}$, similar to the balanced strong cascade.

4. The cascade times of the (larger amplitude) positive waves are longer than their wave periods by the constant factor $\epsilon^{+} / \epsilon^{-} \geq 1$, independent of scale; unlike the imbalanced weak cascade, there is no tendency for the cascade to strengthen at small $\lambda$. When $\epsilon^{+}=\epsilon^{-}$, the turbulence corresponds to the balanced strong cascade of Goldreich \& Sridhar (1995).

\section{NEGLECTED EFFECTS}

We have considered incompressible fluids, whereas astrophysical fluids are not completely incompressible. But compressible MHD fluid motions can be decomposed into four different types of waves: Alfvén, fast, slow, and entropy. Our scalings are applicable to the cascade of the Alfvén waves, which are always incompressible. The effects of compressiblity on the Alfvénic cascade can be neglected if the compressible waves (fast, slow, and entropy) have little effect on the dynamics of the Alfvén waves. Theoretical and numerical investigations have shown that, indeed, the Alfvén waves usually evolve independently of the compressible modes (Lithwick \& Goldreich 2001; Maron \& Goldreich 2001; Cho \& Lazarian 2003).

It has recently been argued that in balanced MHD turbulence, the velocity and magnetic fields tend to align ("dynamical alignment"), and that this alignment can drastically modify the spectrum (Boldyrev 2005; Beresnyak \& Lazarian 2006). Boldyrev (2005) suggests that simulations of MHD turbulence that yield spectra flatter than Kolmogorov support the alignment hypothesis. But observations of solar wind turbulence show spectra that are remarkably consistent with Kolmogorov (e.g., Horbury et al. 2005). So it is not clear whether the flat spectra seen in simulations are due to dynamical alignment or whether they result from the simulations' limited dynamic range. Since dynamical alignment is still not well understood even in the balanced case, we neglect it for now.

\section{APPENDIX}

\section{BACK-REACTION AND THE CORRELATION TIME OF THE NEGATIVE WAVES}

Let $\tau_{\lambda}^{+} \sim \Lambda_{\lambda}^{\prime} / V_{\mathrm{A}}$ denote the timescale over which $w_{\lambda}^{+}$varies at fixed $z^{\prime}$. Below we prove (1) that the correlation time of the negative waves, at fixed $z^{\prime}$, is $\tau_{\text {corr }, \lambda}^{-} \sim \tau_{\lambda}^{+}$and (2) that $\Lambda_{\lambda}^{\prime} / \Lambda_{\lambda} \sim \epsilon^{+} / \epsilon^{-}$.

During the time interval $\tau_{\lambda_{*}}^{+} \sim \Lambda_{\lambda_{*}}^{\prime} / V_{\mathrm{A}}$, negative waves cascade from transverse scales $\lambda_{\max }$ to $\lambda_{*}$. We approximate this cascade as taking place in discrete steps of duration $\tau_{\lambda}^{-} \sim \Lambda_{\lambda} / V_{\mathrm{A}}$, in each of which $\lambda$ decreases by a constant factor of order unity. Thus $\lambda_{\text {max }}$ is related to $\lambda_{*}$ by

$$
\int_{\lambda_{*}}^{\lambda_{\max }} \frac{d \lambda}{\lambda} \Lambda_{\lambda} \sim \Lambda_{\lambda_{*}}^{\prime}
$$

If $\Lambda_{\lambda}^{\prime}$ increases with $\lambda$, equation (A1) implies that

$$
\Lambda_{\lambda_{*}}^{\prime} \sim \Lambda_{\lambda_{\max }}
$$

most of the cascade time is spent near scales $\sim \lambda_{\max }$. 
The positive waves encountered by negative waves, at the same $z^{\prime}$ and $\lambda$ but separated in time by $\tau_{\lambda_{*}}^{+}$, differ by $\delta w_{\lambda}^{+} / w_{\lambda}^{+} \sim \Lambda_{\lambda_{*}}^{\prime} / \Lambda_{\lambda}^{\prime}$. Thus, over each step of the cascade from $\lambda_{\max }$ to $\lambda_{*}$,

$$
\delta w_{\lambda}^{-} \sim \frac{\delta w_{\lambda}^{+}}{\lambda} \frac{\Lambda_{\lambda}}{V_{\mathrm{A}}} w_{\lambda}^{-} \sim \frac{\Lambda_{\lambda_{*}}^{\prime}}{\Lambda_{\lambda}^{\prime}} w_{\lambda}^{-},
$$

where we have used $\chi_{\lambda}^{+} \sim w_{\lambda}^{+} \Lambda_{\lambda} / V_{\mathrm{A}} \lambda \sim 1$. Then the mean square fractional variation of $w_{\lambda_{*}}^{-}$accumulated during the entire cascade amounts to

$$
\left(\frac{\delta w_{\lambda_{*}}^{-}}{w_{\lambda_{*}}^{-}}\right)^{2} \sim \int_{\lambda_{*}}^{\lambda_{\max }} \frac{d \lambda}{\lambda}\left(\frac{\Lambda_{\lambda_{*}}^{\prime}}{\Lambda_{\lambda}^{\prime}}\right)^{2}\left(\frac{w_{\lambda}^{-}}{w_{\lambda_{*}}^{-}}\right)^{2} .
$$

Provided $\Lambda_{\lambda}^{\prime} / w_{\lambda}^{-}$increases with $\lambda$,

$$
\delta w_{\lambda_{*}}^{-} / w_{\lambda_{*}}^{-} \sim 1
$$

Although most of the cascade time is spent near $\lambda_{\max }$ (see eq. [A2]), the accumulated change in $w_{\lambda_{*}}^{-}$comes from scales near $\lambda_{*}$. Moreover, since $w_{\lambda_{*}}^{-}$varies by order unity, at fixed $z^{\prime}$, during the time interval $\tau_{\lambda}^{+}$, the correlation time of the negative waves is

$$
\tau_{\text {corr }, \lambda}^{-} \sim \tau_{\lambda}^{+} \sim \lambda / w_{\lambda}^{-},
$$

which proves item 1 . To prove item 2 , we note that

$$
\frac{\Lambda_{\lambda}^{\prime}}{\Lambda_{\lambda}} \sim \frac{\tau_{\lambda}^{+}}{\tau_{\lambda}^{-}} \sim \frac{w_{\lambda}^{+}}{w_{\lambda}^{-}} \sim \frac{\epsilon^{+}}{\epsilon^{-}} \geq 1
$$

where we have used equations (A6) and (14)-(15).

${ }^{9}$ We reserve the symbol $\delta$ to denote differences accrued over the time interval $\tau_{\lambda_{*}}^{+}$.

Beresnyak, A., \& Lazarian, A. 2006, ApJ, 640, L175

Biskamp, D. 2003, Magnetohydrodynamic Turbulence (Cambridge: Cambridge Univ. Press)

Biskamp, D., \& Müller, W.-C. 2000, Phys. Plasmas, 7, 4889

Boldyrev, S. 2005, ApJ, 626, L37

Cho, J., \& Lazarian, A. 2003, MNRAS, 345, 325

Cho, J., Lazarian, A., \& Vishniac, E. T. 2002, ApJ, 564, 291

Cho, J., \& Vishniac, E. T. 2000, ApJ, 539, 273

Galtier, S., Nazarenko, S. V., Newell, A. C., \& Pouquet, A. 2000, J. Plasma Phys., 63, 447 2002, ApJ, 564, L49

Galtier, S., Pouquet, A., \& Mangeney, A. 2005, Phys. Plasmas, 12, No. 092310 Goldreich, P., \& Sridhar, S. 1995, ApJ, 438, 763

$$
\text { . 1997, ApJ, 485, } 680
$$

Horbury, T. S. 1999, in Plasma Turbulence and Energetic Particles in Astrophysics, ed. M. Ostrowski \& R. Schlickeiser (Cracow: Obs. Astron., Uniwersytet Jagielloński), 115

Horbury, T. S., Forman, M. A., \& Oughton, S. 2005, Plasma Phys. Controlled Fusion, 47, B703

Iroshnikov, P. S. 1963, AZh, 40, 742 (English transl. Soviet Astron.-AJ, 7, 566 [1964])

\section{REFERENCES}

Kraichnan, R. H. 1965, Phys. Fluids, 8, 1385

Kulsrud, R. M. 2005, Plasma Physics for Astrophysics (Princeton: Princeton Univ. Press)

Lithwick, Y., \& Goldreich, P. 2001, ApJ, 562, 279

- 2003, ApJ, 582, 1220

Maron, J., \& Goldreich, P. 2001, ApJ, 554, 1175

Müller, W.-C., Biskamp, D., \& Grappin, R. 2003, Phys. Rev. E, 67, No. 066302

Müller, W.-C., \& Grappin, R. 2005, Phys. Rev. Lett., 95, No. 114502

Ng, C. S., \& Bhattacharjee, A. 1996, ApJ, 465, 845

Politano, H., \& Pouquet, A. 1998a, Phys. Rev. E, 57, 21 1998b, Geophys. Res. Lett., 25, 273

Schekochihin, A., \& Cowley, S. C. 2007, in Magnetohydrodynamics: Historical Evolution and Trends, ed. S. Molokov, R. Moreau, \& H. K. Moffatt (Berlin: Springer), in press (astro-ph/0507686)

Shebalin, J. V., Matthaeus, W. H., \& Montgomery, D. 1983, J. Plasma Phys., 29,525

Verma, M. K. 2004, Phys. Rep., 401, 229

Verma, M. K., Roberts, D. A., Goldstein, M. L., Ghosh, S., \& Stribling, W. T. 1996, J. Geophys. Res., 101, 21619 